\title{
Ureteral Stent Induced Urinary Tract Infection and Microbial Inconsistency Between Bladder and Renal Pelvis
}

\author{
Useok Choi, Eun Jae Kim, Don Hee Lyu, Bong Hee Park, Hong Chung ${ }^{1}$, Chang Hee Han, Sangrak Bae \\ Department of Urology, The Catholic University of Korea, Uijeongbu St. Marys Hospital, Uijeongbu, 'Department of Urology, Konkuk \\ University School of Medicine, Chungju, Korea
}

Purpose: This study examined the effects of long-term ureteral stent (US) on urinary tract infection (UTI) by bacterial migration and bacterial colonization.

Materials and Methods: From March 2014 to August 2021, 64 patients who underwent US for more than six months were enrolled in this study. When the US was inserted for the first time and replaced regularly, urine was collected from the bladder and renal pelvis and cultured. Patients' the characteristics, and differences in pathogens related to US infection were analyzed.

Results: The mean age was 66.4 years, and the mean duration of indwelling was 37.5 months. There were 38 cases (59.3\%) by malignant and 26 cases (40.6\%) by benign causes. UTI occurred in 33 cases (53\%); 16 cases (25\%) were hospitalized for febrile UTI. After the initial US insertion, bacteria were identified in the bladder 285 days later and in the renal pelvis after 619 days. The bladder culture was positive in 10 of the 33 patients, and renal pelvis alone was positive in nine of the 33 patients. The same strain was confirmed in the bladder and renal pelvis in 12 cases (18.8\%), and it took a mean of 5.8 months \pm 6.32 (standard deviation) for the upward migration of bacteria.

Conclusions: UTIS occur in approximately $50 \%$ of patients with long-term US indwelling. The probability of culturing the same strain by a US was approximately $18.8 \%$, and the role of pathogen transmission of a US does not appear to be significant. In addition, renal pelvis culture would be helpful in cases of failed bladder antibiotics susceptibility.

Keywords: Urinary tract infections; Stents; Urinary tract

Copyright (C) 2021, Korean Association of Urogenital Tract Infection and Inflammation.

(1) (\$) This is an open access article distributed under the terms of the Creative Commons Attribution Non-Commercial License (http://creativecommons.org/licenses/by-nc/4.0) which permits unrestricted non-commercial use, distribution, and reproduction in any medium, provided the original work is properly cited.
Received: 15 October, 2021

Revised: 8 November, 2021

Accepted: 19 November, 2021
Correspondence to: Sangrak Bae

(iD) https://orcid.org/0000-0002-8364-704X

Department of Urology, The Catholic University of Korea, Uijeongbu St. Mary's Hospital, 271 Cheonboro, Uijeongbu 11765 , Korea

Tel: +82-31-820-5354, Fax: +82-31-847-6133

E-mail: robinbae97@catholic.ac.kr

\section{INTRODUCTION}

Ureteral stents (or double J stent, double pigtail stent, US) are used widely in various urological areas [1]. They are inserted to solve obstructions of the urine stream by ureter obstructions, strictures, and stones. They are also used for various urolithiasis and endourologic surgeries or procedure, such as retrograde intrarenal surgery, flexible ureteroscopic surgery, and percutaneous nephrolithotomy, and to help prevent stricture and recover the surgical site after ureter-related surgery. In the case of semi-rigid ureteroscopic surgery for urolithiasis, a large study reported that a postoperative US is performed in approximately $81 \%$ of all patients [2]. In the case of a malignant ureteral obstruction, a US is inserted to improve the quality of life or prevent complications [3]. On the other hand, a US can 
cause various side effects, such as pain [4], urinary discomfort [5], hematuria, urinary tract infection (UTI) [6-9], and the occurrence of stones [10]. Therefore, various opinions exist on the necessity of a ureter stent [11]. Long-term indwelling in the body can cause problems with biofilm formation, recurrent UTIs, and even a life-threatening status caused by urosepsis.

In general, lower UTIs, such as acute cystitis, have an incidence of 0.5 to 0.7 person-year [12]; the annual incidence of acute pyelonephritis (APN) in Korea is 39.1 per 10,000 persons [13]. Infective complications associated with a US include uncomplicated/complicated cystitis, including acute cystitis, APN, asymptomatic bacteriuria, and recurrent UTI $[8,14,15]$.

Urine flows in one direction from the kidney to the bladder, and an anti-reflux system located at the junction of the bladder and ureter prevents urine reflux. On the other hand, when a US is inserted, the urine from the lower urinary tract can be delivered to the upper urinary tract by bypassing the anti-reflux system. Infected urine from the lower urinary tract can also be transferred to the upper urinary tract [11]. A recent study cautioned that the insertion of a US in a malignant tumor could cause an upper ureter metastasis by transferring the urothelial cells from the urinary bladder to the ureter [16]. Furthermore, a few reports on the effects of long-term US indwelling reported the following: 1) complications due to insertion and indwelling itself, 2) risks according to the period, 3) possibility of complications, and 4) bacterial characteristics of UTIs that are likely to occur during long-term storage.

This study examined the association between US-related infectious complications and causative bacteria in long-term US indwelling patients, differences in bacterial characteristics found in the bladder and renal pelvis in patients with US implantation, and their clinical significance.

\section{MATERIALS AND METHODS}

\section{Study Design}

Institutional ethics review was sought and the study was approved by The Catholic University of Korea, Institutional Review Board (Approval no. UC22RISI0001). Because only medical records were used, informed consent was not obtained. From March 2014 to August 2021, 64 patients who underwent a long-term US for more than six months that was changed every 12 weeks were included. US was inserted to prevent complications from hydronephrosis or urinary tract narrowing caused by malignant and benign diseases, such as malignant tumors or ureteral stricture. Periodic changes of a US are required in cases of prior radiation therapy to the abdomen and pelvis, if the patient or family refuses anesthesia, and if conservative treatment is desired. All patients were inserted with a Percuflex stent (Boston Scientific, Marlborough, MA, USA) of different lengths and diameters. In addition to the patients' characteristics, the reason for the ureteral stent, the level and side of ureteral obstruction, and the diameter and length of the US. Comorbidities, history of abdominopelvic surgery, whether chemotherapy or radiation therapy is performed, and indwelling Foley or suprapubic catheter were also evaluated.

\section{Ureteral Stent Insertion and Routine Change}

US insertion was performed via the antegrade or retrograde method at the radiology and urology department, respectively. In the case of US exchange, it was replaced under local anesthesia in the operating room by endoscopy. Urine in the bladder was collected using a nelaton catheter (Fig. 1A), and a unilateral/bilateral 5F ureteral catheter was used to collect urine from the renal pelvis (Fig. 1B). Specimens were collected using different $5 \mathrm{Fr}-$ open-end ureteral catheters in the case of patients with a bilateral US. The urine samples were labeled to avoid confusion in the bladder and left/right renal pelvis samples and clearly determine the presence and type of bacteria confirmed through urine culture and the antibiotics susceptibility results. In cases where drainage was difficult due to the absence of hydronephrosis, regurged fluid after
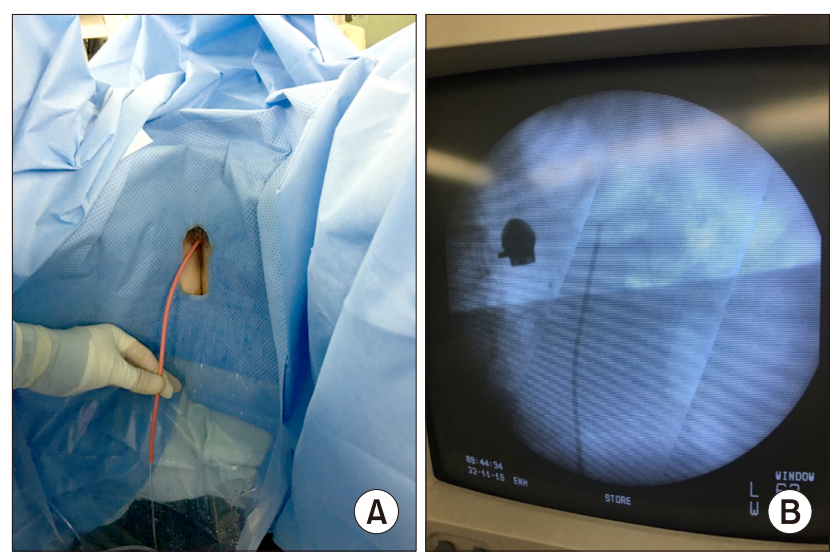

Fig. 1. Method of collecting urine culture specimen according to organs. (A) Urine sample collected by a nelaton catheter. (B) Urine sample collected by a plain ureteral catheter for a renal pelvis specimen. 
injecting $5 \mathrm{ml}$ of normal saline was collected. The duration from US insertion to the date the bacteria were identified was defined as the 'period of bacterial development'. The duration of bacterial migration from the bladder to the renal pelvis was defined as the 'transmission period'.

\section{RESULTS}

\section{Baseline Patients' Characteristics}

The mean age of patients was 66.6 years, and the mean body mass index was $23.4 \mathrm{~kg} / \mathrm{m}^{2}$. There were 13 males (20.3\%) and 51 females (79.3\%). Table 1 lists the baseline patient characteristics. The mean duration of US indwelling was 37.5 months. A US was inserted more for malignancies than benign diseases (each malignancy in 37 cases [58\%] and benign disease in 27 cases [42\%]). Among the malignancies, gynecological cancer, such as cervical cancer, uterus cancer, ovarian cancer, and endometrial cancer, were the most common in 19 cases, followed by colorectal cancer in nine cases, urologic cancer in four cases, and other malignancies

Table 1. Baseline characteristics of the patients

\begin{tabular}{|c|c|}
\hline Variable & Value \\
\hline Mean age (yr) & 66.6 \\
\hline Mean BMI $\left(\mathrm{kg} / \mathrm{m}^{2}\right)$ & 23.4 \\
\hline \multicolumn{2}{|l|}{ Sex } \\
\hline Male & 13 \\
\hline Female & 51 \\
\hline \multicolumn{2}{|l|}{ Side } \\
\hline Both & 19 \\
\hline Left & 27 \\
\hline Right & 18 \\
\hline \multicolumn{2}{|l|}{ Level of obstruction } \\
\hline Upper & 23 \\
\hline Mid & 6 \\
\hline Lower & 35 \\
\hline \multicolumn{2}{|l|}{ Cause of insertion } \\
\hline Malignancy & 38 \\
\hline Benign & 26 \\
\hline \multicolumn{2}{|c|}{ Abdominopelvic surgery } \\
\hline Yes & 40 \\
\hline No & 24 \\
\hline \multicolumn{2}{|l|}{ Chemotherapy } \\
\hline Yes & 37 \\
\hline No & 27 \\
\hline \multicolumn{2}{|l|}{ Radiation } \\
\hline Yes & 32 \\
\hline No & 32 \\
\hline \multicolumn{2}{|c|}{ Foley catheter indwelling } \\
\hline Yes & 15 \\
\hline No & 49 \\
\hline \multicolumn{2}{|c|}{ Admission for febrile UTI } \\
\hline Yes & 16 \\
\hline No & 48 \\
\hline
\end{tabular}

BMI: body mass index, UTI: urinary tract infection. in five cases.

\section{Ureteral Stent-Related Complications}

One or more types of bacteria were identified in a bladder specimen or renal pelvis specimen in 47 cases (73.4\%). Among them, 33 patients (51.6\%) complained of symptoms accompanying lower urinary tract infection, such as lower abdominal/suprapubic area pain, dysuria, residual urine sensation, and frequency. Therefore, 33 patients were administered oral or IV antibiotics. Sixteen patients, one-quarter of all patients (25\%), were hospitalized for urinary tract infections with fever.

Hematuria was identified in all patients. Among the abnormal findings during the US exchange procedure, nine patients had lumen obstruction, so the US was replaced. Encrustation of the US occurred in the proximal and distal parts; discoloration occurred on the US surface in 33 patients.

Among the patients hospitalized for febrile UTI, seven patients (10.9\%) were multiply hospitalized, and four patients (6.3\%) required intensive care because of septic conditions. Four of the 16 hospitalized patients (25\%) had different types of bacteria between the bladder and renal pelvis. In these patients, treatment according to the antibiotic susceptibility results from the bladder urine did not improve the clinical course. The antibiotic susceptibility test of urine from the renal pelvis was essential for successful treatment (Table 2).

The most serious complication occurred in a 55-year-old female patient with a ureter stricture after undergoing a radical hysterectomy and chemotherapy with radiation for cervical cancer. The patient was hospitalized three times within six months for recurrent acute pyelonephritis on a blood test and imaging tests, such as a computed tomography scan. A life-threatening aorto-ureteral fistula occurred at the time of removing the US. The bleeding was controlled by inserting an aortic stent through vascular intervention.

Regular US exchange was discontinued in 28 patients

Table 2. Differences in bacteria identified in the bladder and renal pelvis of patients who failed initial treatment

\begin{tabular}{cll}
\hline Case & \multicolumn{1}{c}{ Bladder } & \multicolumn{1}{c}{ Renal pelvis } \\
\hline 1 & ESBL(-) Escherichia coli & ESBL(+) Klebsiella \\
2 & Escherichia coli & Enterococcus faecium \\
3 & ESBL(-) Klebsiella & ESBL(+) Escherichia coli \\
4 & Pseudomonas & Vancomycin sensitive \\
& & Enterococcus
\end{tabular}

ESBL: extended spectrum beta-lactamase. 
Table 3. Number of strains identified in the bladder and renal pelvis

\begin{tabular}{|c|c|c|c|c|c|}
\hline & Bladder & Number & & Renal pelvis & Number \\
\hline \multicolumn{2}{|c|}{ Escherichia coli } & 11 & \multicolumn{2}{|c|}{ Escherichia coli } & 10 \\
\hline \multicolumn{2}{|c|}{ Enterococcus faecalis } & 6 & \multicolumn{2}{|c|}{ Enterococcus faecalis } & 2 \\
\hline \multicolumn{2}{|c|}{ Enterococcus faecium } & 2 & \multicolumn{2}{|c|}{ Enterococcus faecium } & 5 \\
\hline \multicolumn{2}{|c|}{ klebsiella pneumoniae } & 5 & \multicolumn{2}{|c|}{ Klebsiella pneumoniae } & 2 \\
\hline \multicolumn{2}{|c|}{ Pseudomonas aerusinosa } & 6 & \multicolumn{2}{|c|}{ Pseudomonas aerusinosa } & 4 \\
\hline \multicolumn{2}{|c|}{ Proteus mirabilis } & 3 & \multicolumn{2}{|c|}{ Proteus mirabilis } & 3 \\
\hline \multirow[t]{5}{*}{ Etc. } & Enterococcus cloacae & 1 & Etc. & beta-hemolytic Streptococcus & 2 \\
\hline & Acinetobacter baumanni & 1 & & Citrobacter freundii & 2 \\
\hline & beta-hemolytic Streptococcus & 1 & & Enterococcus cloacae & 1 \\
\hline & Providencia situartii & 1 & & Providencia situartii & 1 \\
\hline & & & & Corynebacterium & 1 \\
\hline Total & & 37 & Total & & 33 \\
\hline
\end{tabular}

(43.8\%) for the following reasons: five cases (17.9\%) for complications, 13 cases (46.4\%) for patient's death, and 10 cases (35.7\%) for follow-up loss.

\section{Bacterial Characteristics}

On the first US insertion, bacteria in the bladder were cultured in 10 patients (15.6\%). After stent insertion, bacteria were identified in the bladder a mean 9.5 months later and in the renal pelvis after 20.6 months. Table 3 lists the bacteria identified in urine culture from the bladder and renal pelvis. The most common pathogen was Escherichia coli in the bladder and renal pelvis. As another feature, Pseudomonas and Enterococcus faecalis strains were identified relatively frequently in the bladder, but Enterococcus faecium was more frequently in the renal pelvis.

The same bacteria were identified in the bladder and renal pelvis in 12 patients (18.8\%). The bacteria were first identified in the bladder, and the same bacteria were identified in the renal pelvis after an average of approximately 5.8 months. The antibiotic susceptibility results were the same in these patients, confirming that it took about six months for transmission from the bladder to the renal pelvis. In the remaining 52 patients, bacteria grew only in the bladder in 10 patients (15.6\%) and only in the renal pelvis in nine patients (14.1\%).

\section{DISCUSSION}

A US is the most widely used tube-like drainage device used alongside drainage catheters in urologic procedures, and has been used in many areas, such as obstructive nephropathy, pain control, and the prevention of postoperative complications [1,17-19]. Since a US is also a foreign body, long-term indwelling in the human body can cause various problems, including infection complications, so it should be inserted only when necessary. It should be removed when the medical indication is resolved to prevent complications [1,5,20,21]. Commonly, US is inserted after ureteroureterostomy, ureteroneocystostomy, ureterolithomy, and pyeloplasty.

Although a US is inserted for therapeutic purposes, patients experience various discomforts or adverse events during the US indwelling period. In particular, infectious complications $[6,8,9,11]$ can occur. In the case of infectious complications, the US itself becomes a site of bacterial colonization and biofilm formation, which is a source of urinary tract infections $[6,8,9,11,15,20]$. In most cases, this risk can increase with prolonged indwelling.

In the case of a ureteral stent, a US acts as a bypass tract in the anti-reflux system. Urine in the bladder can easily reflux into the ureter or renal pelvis. In addition to urinating, if the urinate is held for a long time, the pressure in the bladder increases, causing the urine from the bladder to flow back into the kidneys. The refluxed urine increases the pressure and dilates the ureter or renal pelvis, causing flank pain. Therefore, the bacterial infection in the bladder is transferred easily to the renal pelvis, which becomes a risk factor, such as pyelonephritis, and increases the risk of febrile UTI. As the US induces reflux and acts as a pathway for bacteria to move, it would be natural for bacteria in the bladder to be confirmed in the renal pelvis. Surprisingly, although US was expected to transfer bacteria from the bladder to the kidney easily, the same bacteria were identified only in $18.8 \%$, and it took a mean of 5.8 months.

In addition, the overall incidence of UTI and febrile UTI that required hospitalization showed a higher incidence and prevalence of UTI than general patient groups, such as 0.5-0.7 
person-years in uncomplicated/complicated UTI [12] and an annual incidence of APN in Korea [22]. The long-term indwelling of a US may be one of the risk factors for various urinary tract infections compared to the general population $[6,8,9]$. Urinary tract infections occurred at a high rate in this study because more than $50 \%$ of long-term indwelling patients had urinary tract infections, and the rate of febrile urinary tract infections was $25 \%$.

Different colonization between the bladder and the renal pelvis was reported in studies of patients with renal calculi $[23,24]$. A urine sample was obtained from the renal pelvis during renal stone surgery, and a urine culture test and antibiotic susceptibility test were performed. The use of antibiotics based on the results of renal pelvis samples could effectively respond to various infection-related complications and sepsis that occur after surgery.

As urine reflux is caused by the transfer of urine from the lower urinary tract to the upper urinary tract through the US, tumor cells can be transferred, or bacteria can migrate. Considerable efforts have been made to prevent an event that bypasses the anti-reflux system from occurring and prevent disease transmission, even though it passes through the US [25]. In addition to disease transmission, various attempts have been made to prevent various complications related to the US, such as a change in the material or shape, and apply a drug or chemical agent coating to the surface $[17,26]$. In addition, many studies have evaluated the advantages of metal stents in patients undergoing long-term ureteral stent indwelling $[27,28]$. In the case of a metal stent, frequent replacement can be avoided, and related medical costs can be reduced. In addition, stronger resistance to the pressure from external compression than the Double J US can be observed. On the other hand, the use of metal stents was not considered in this study because of the disadvantages, such as the formation of stones in the lumen and various complications [29].

Although not a limitation, the number of target patients was small due to the characteristics of the disease and patient group. Significant results are expected if a multicenter study is conducted with more patients.

\section{CONCLUSIONS}

The long-term indwelling of a US had an approximately 50\% higher tendency for urinary tract infection. By indwelling the US, the ratio of delivering bacteria in the bladder to the renal pelvis was approximately $20 \%$, and it took almost six months. The ureteral stent itself is not used unconditionally as a passageway for bacteria to spread infection from the lower urinary tract to the upper urinary tract. In addition, the collection of urine samples from the renal pelvis can provide therapeutic benefits in cases of a febrile UTI with an unsuccessful initial empirical treatment.

\section{CONFLICT OF INTEREST}

No potential conflict of interest relevant to this article was reported.

\section{ACKNOWLEDGEMENTS}

This work was supported by The Catholic University of Korea, Uijeongbu St. Mary's Hospital, Clinical Medicine Research Institute.

\section{AUTHOR CONTRIBUTIONS}

U.C., E.J.K., D.H.L., S.B. participated in data collection and U.C. and S.B. wrote the manuscript. C.H.H. and S.B. participated in the study design and performed the statistical analysis. B.H.P. and H.C. participated in the study design and coordination and helped to draft the manuscript. All authors read and approved the final manuscript.

\section{ORCID}

Useok Choi, https://orcid.org/0000-0002-1203-2312

Eun Jae Kim, https://orcid.org/0000-0002-9888-6473

Don Hee Lyu, https://orcid.org/0000-0003-0441-2283

Bong Hee Park, https://orcid.org/0000-0002-7686-3094

Hong Chung, https://orcid.org/0000-0002-0151-4965

Chang Hee Han, https://orcid.org/0000-0001-7427-5793

Sangrak Bae, https://orcid.org/0000-0002-8364-704X

\section{REFERENCES}

1. Sali GM, Joshi HB. Ureteric stents: overview of current clinical applications and economic implications. Int J Urol 2020;27: 7-15.

2. de la Rosette J, Denstedt J, Geavlete P, Keeley F, Matsuda T, Pearle M, et al.; CROES URS Study Group. The clinical research office of the Endourological Society ureteroscopy global study: 
indications, complications, and outcomes in 11,885 patients. J Endourol 2014;28:131-9.

3. Tabib C, Nethala D, Kozel Z, Okeke Z. Management and treatment options when facing malignant ureteral obstruction. Int J Urol 2020;27:591-8.

4. Koprowski C, Kim C, Modi PK, Elsamra SE. Ureteral stent-associated pain: a review. J Endourol 2016;30:744-53.

5. Fischer KM, Louie M, Mucksavage P. Ureteral stent discomfort and its management. Curr Urol Rep 2018;19:64.

6. Akay AF, Aflay U, Gedik A, Sahin H, Bircan MK. Risk factors for lower urinary tract infection and bacterial stent colonization in patients with a double J ureteral stent. Int Urol Nephrol 2007;39:95-8.

7. Al-Ghazo MA, Ghalayini IF, Matani YS, El-Radaideh KM, Haddad HI. The risk of bacteriuria and ureteric stent colonization in immune-compromised patients with double J stent insertion. Int Urol Nephrol 2010;42:343-7.

8. Joshi R, Singh DR, Sharma S. Lower urinary tract infection and bacterial colonization in patient with double J ureteral stent. J Nepal Health Res Counc 2011;9:165-8.

9. Liaw A, Knudsen B. Urinary tract infections associated with ureteral stents: a review. Arch Esp Urol 2016;69:479-84.

10. Kram W, Buchholz N, Hakenberg OW. Ureteral stent encrustation. Pathophysiology. Arch Esp Urol 2016;69:485-93.

11. Scotland KB, Lo J, Grgic T, Lange D. Ureteral stent-associated infection and sepsis: pathogenesis and prevention: a review. Biofouling 2019;35:117-27.

12. Gupta K, Grigoryan L, Trautner B. Urinary tract infection. Ann Intern Med 2017;167:ITC49-64.

13. Johnson JR, Russo TA. Acute pyelonephritis in adults. N Engl J Med 2018;378:48-59.

14. Tsukanov AY, Akhmetov DS, Blesman Al, Rogachev EA. [The impact of ureteral stent surface on encrustation and biofilm formation]. Urologiia 2018;2:40-5. Russian.

15. Zhang JM, Liu J, Wang K, Zhang X, Zhao T, Luo HM. Observations of bacterial biofilm on ureteral stent and studies on the distribution of pathogenic bacteria and drug resistance. Urol Int 2018;101:320-6.

16. Sountoulides P, Pyrgidis N, Brookman-May S, Mykoniatis I, Karasavvidis T, Hatzichristou D. Does ureteral stenting increase the risk of metachronous upper tract urothelial carcinoma in patients with bladder tumors? A systematic review and meta-analysis. J Urol 2021;205:956-66.

17. Chew BH, Lange D. Advances in ureteral stent development.
Curr Opin Urol 2016;26:277-82.

18. Chew BH, Seitz C. Impact of ureteral stenting in ureteroscopy. Curr Opin Urol 2016;26:76-80.

19. Chugh S, Pietropaolo A, Montanari E, Sarica K, Somani BK. Predictors of urinary infections and urosepsis after ureteroscopy for stone disease: a systematic review from EAU section of urolithiasis (EULIS). Curr Urol Rep 2020;21:16.

20. Lange D, Bidnur S, Hoag N, Chew BH. Ureteral stent-associated complications--where we are and where we are going. Nat Rev Urol 2015;12:17-25.

21. Lundeen $\mathrm{CJ}$, Forbes $\mathrm{CM}$, Wong VKF, Lange $\mathrm{D}$, Chew $\mathrm{BH}$. Ureteral stents: the good the bad and the ugly. Curr Opin Urol 2020;30:166-70.

22. Kim B, Myung R, Kim J, Lee MJ, Pai H. Descriptive epidemiology of acute pyelonephritis in Korea, 2010-2014: population-based study. J Korean Med Sci 2018;33:e310.

23. Karsiyakali N, Yucetas U, Karatas A, Karabay E, Okucu E, Erkan E. Renal pelvis urine Gram stain as a traditional, but new marker in predicting postoperative fever and stone culture positivity in percutaneous nephrolithotomy: an observational, prospective, non-randomized cohort study. World J Urol 2021;39:2135-46.

24. Yoshida S, Takazawa R, Uchida Y, Kohno Y, Waseda Y, Tsujii T. The significance of intraoperative renal pelvic urine and stone cultures for patients at a high risk of post-ureteroscopy systemic inflammatory response syndrome. Urolithiasis 2019;47:533-40.

25. Soria F, Morcillo E, de la Cruz JE, Serrano A, Estebanez J, Sanz JL, et al. Antireflux ureteral stent proof of concept assessment after minimally invasive treatment of obstructive uropathy in animal model. Arch Esp Urol 2018;71:607-13.

26. Forbes $\mathrm{C}$, Scotland KB, Lange D, Chew BH. Innovations in ureteral stent technology. Urol Clin North Am 2019;46:245-55.

27. Benson AD, Taylor ER, Schwartz BF. Metal ureteral stent for benign and malignant ureteral obstruction. J Urol 2011;185: 2217-22.

28. Sampogna G, Grasso A, Montanari E. Expandable metallic ureteral stent: indications and results. Minerva Urol Nefrol 2018;70:275-85.

29. Kallidonis P, Kotsiris D, Sanguedolce F, Ntasiotis P, Liatsikos E, Papatsoris A; Young Academic Urologists of the European Association of Urology - Endourology, Urolithiasis Working Party. The effectiveness of ureteric metal stents in malignant ureteric obstructions: a systematic review. Arab J Urol 2017;15:280-8. 